\title{
STABLE PATTERNS \\ IN A VISCOUS DIFFUSION EQUATION
}

\author{
A. NOVICK-COHEN AND R. L. PEGO
}

\begin{abstract}
We consider a pseudoparabolic regularization of a forward-backward nonlinear diffusion equation $u_{t}=\Delta\left(f(u)+\nu u_{t}\right)$, motivated by the problem of phase separation in a viscous binary mixture. The function $f$ is nonmonotone, so there are discontinuous steady state solutions corresponding to arbitrary arrangements of phases. We find that any bounded measurable steady state solution $u(x)$ satisfying $f(u)=$ constant, $f^{\prime}(u(x))>0$ a.e. is dynamically stable to perturbations in the sense of convergence in measure. In particular, smooth solutions may achieve discontinuous asymptotic states. Furthermore, stable states need not correspond to absolute minimizers of free energy, thus violating Gibbs' principle of stability for phase mixtures.
\end{abstract}

\section{INTRODUCTION}

This paper is concerned with the study of the equation

$$
u_{t}=\Delta\left(f(u)+\nu u_{t}\right), \quad x \in \Omega, t \in \mathbf{R},
$$

with boundary condition

$$
n \cdot \nabla\left(f(u)+\nu u_{t}\right)=0, \quad x \in \partial \Omega, t \in \mathbf{R},
$$

and initial condition

$$
u(x, 0)=u_{0}(x), \quad x \in \Omega .
$$

Here $f$ is like a nonmonotonic cubic as in Figure 1; precise hypotheses are specified in (F1) in $\S 3$ below. $\Omega$ is a bounded, connected domain in $\mathbf{R}^{N}$ with smooth boundary and outward unit normal $n$, and $\nu>0$ is a constant. Equation (1.1) arises as a regularization of the forward-backward nonlinear diffusion equation $u_{t}=\Delta f(u)$, a regularization different from that incorporated in the Cahn-Hilliard equation

$$
u_{t}=\Delta(f(u)-\kappa \Delta u) .
$$

The Cahn-Hilliard equation was introduced by Cahn [C] to model the isothermal phase separation of a binary mixture quenched into an unstable homogeneous state. It is based on the near-equilibrium dynamic principle that material

Received by the editors March 31, 1989.

1980 Mathematics Subject Classification (1985 Revision). Primary 35K70, 35B35, 35K55, $80 \mathrm{~A} 15,82 \mathrm{~A} 25,35 \mathrm{Q} 99$.

Key words and phrases. Pattern formation, viscosity, phase separation, dissipative system, pseudoparabolic, nonlinear stability. 


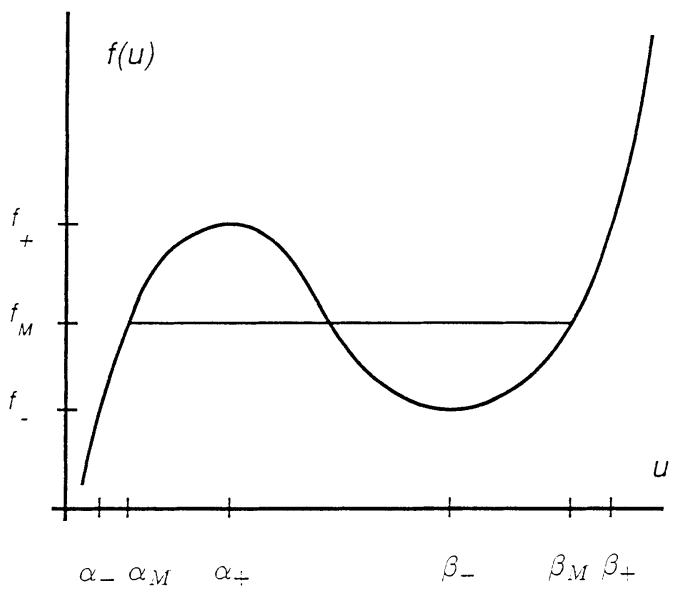

FIGURE 1

flux should be proportional to the gradient of chemical potential. In terms of the concentration $u(x, t)$ of one species, the chemical potential $\mu=f(u)-\kappa \Delta u$ arises as the functional derivative of a free energy functional which decreases in time for solutions of (1.4), namely

$$
I(u)=\int_{\Omega}\left(F(u)+\frac{1}{2} \kappa|\nabla u|^{2}\right), \quad \text { where } F^{\prime}(u)=f(u) .
$$

Seeking better quantitative accord with experimental results, some authors have recently proposed modifications to Cahn's model which incorporate outof-equilibrium viscoelastic relaxation effects. Binder, Frisch, and Jäckle [BFJ] proposed a linear model for phase separation by spinodal decomposition when the chemical potential contains an additional integral relaxation term. When the relaxation kernel is a single exponential $\exp (-\gamma t)$, the equation they obtain for the concentration is equivalent to the equation

$$
u_{t t}+\gamma u_{t}=M \Delta\left(\gamma D_{0} u-\gamma K \Delta u+D_{\infty} u_{t}-K \Delta u_{t}\right),
$$

where $M, D_{0}, D_{\infty}$, and $K$ are constants. Stephenson [S] has also obtained a system of equations equivalent to (1.6). Related equations have been proposed by several authors as models of "nonclassical diffusion". The equation

$$
u_{t}+D^{*} u_{t t}=\Delta\left(D u-E \Delta u+\bar{D} u_{t}\right)
$$

appears in the work of Aifantis [A], while equation (1.6) with $K=0$ arises in work of Dürning [Du] and Jäckle and Frisch [JF1, JF2].

Taking $K=0$ and letting $\gamma$ become large in (1.6) with $D_{\infty} / \gamma$ fixed, one obtains a linear equation of the type in (1.1). This linear equation is also mentioned by Aifantis [A] and has been studied by Ting [T1-T3] and Chen and Gurtin [CG]. The term $\Delta u_{t}$ in (1.1) is interpreted as due to viscous relaxation effects, or viscosity. In work in progress, we can also justify an equation like (1.1) based on considering a slow flow limit for equations of motion of a mixture of two fluids with Newtonian viscosity. 
The nonlinear equation (1.1) may therefore be considered as a variant of the Cahn-Hilliard equation with viscous effects. As a model for phase separation by spinodal decomposition, equation (1.1) has the serious flaw that energy due to inhomogeneities or phase boundaries is neglected. It is nevertheless interesting to determine the mathematical consequences of neglecting these effects, to understand what might be expected when their contribution is small. One expects the high-frequency instabilities present in the backward diffusion equation to contribute to the generation of complex patterns in solutions of the nonlinear equation (1.1). We study here the long-time behavior of solutions, to determine whether solutions stabilize to a steady state, what patterns are stable and in what sense.

The results are somewhat unusual for a dissipative system. It is easy to show that for unsteady solutions of (1.1), the energy

$$
I(u)=\int_{\Omega} F(u)
$$

is decreasing in time, while the average concentration

$$
\bar{u}=\int_{\Omega} u /|\Omega|
$$

is constant in time. But the dissipative mechanism is rather weak: it is not easy to prove that solutions stabilize to a steady state as $t$ becomes large. Following the tradition of Gibbs, one nevertheless expects the solution to evolve toward a state which minimizes the energy $I(u)$ subject to the constraint $\bar{u}=$ constant . If $\bar{u}$ lies between the values $\alpha_{M}$ and $\beta_{M}$ indicated in Figure 1 , which are determined by the Maxwell equal-area construction (so $f\left(\alpha_{M}\right)=f\left(\beta_{M}\right)=f_{M}$ and the integral of $f-f_{M}$ over $\left(\alpha_{M}, \beta_{M}\right)$ is zero), it is well known that a state $u(x)$ achieving the absolute minimum must satisfy, at each point $x$,

$$
\text { either } u(x)=\alpha_{M} \text { or } u(x)=\beta_{M} \text {, so } f(u)=f_{M} \text { everywhere. }
$$

It turns out that these expectations are incorrect. To begin with, there are far more steady state solutions of (1.1) which satisfy the constraint on $\bar{u}$ : Any bounded measurable function $u(x)$ satisfying $f(u(x))=$ constant is a timeindependent solution of (1.1), regardless of whether $f=f_{M}$ or not. That is, as long as the chemical potential is (any) constant, phases may be arranged in an arbitrary pattern in space. Moreover, the following results demonstrate that such solutions can be dynamically stable without being constrained by the equal area law.

Theorem 1. Assume that $f$ is $C^{1}$ and that $u_{e}(x)$ is a steady state solultion of (1.1), so that for some constants $f_{e}$ and $f_{*}$ we have

$$
f\left(u_{e}(x)\right)=f_{e}, \quad f^{\prime}\left(u_{e}(x)\right) \geq f_{*}>0 \quad \text { for a.e. } x \text { in } \Omega \text {. }
$$

Then there is a positive constant $\varepsilon>0$ such that if $u(x, t)$ is a solution of (1.1) that satisfies $\left|u(x, 0)-u_{e}(x)\right|<\varepsilon$ for $x$ in $\Omega$ and $\int_{\Omega}\left(u(x, 0)-u_{e}(x)\right) d x=0$, then $u(x, t)$ converges exponentially fast to $u_{e}(x)$ uniformly in $x$ as $t$ grows. 
Theorem 2. Suppose that $f$ satisfies (F1) and is $C^{1}$, with $f^{\prime}(u)<0$ for $u \in$ $\left(\alpha_{+}, \beta_{-}\right)$, that $u(x, t)$ is a solution of $(1.1)$ such that $|u(x, 0)|$ is bounded, and that $u_{e}(x)$ satisfies (1.9). Suppose that $\left|u(x, 0)-u_{e}(x)\right|>\varepsilon_{1}$ only for $x$ in a set $\Omega_{\varepsilon}$ of measure less than $\varepsilon$. Then, if $\varepsilon$ and $\varepsilon_{1}$ are sufficiently small, it follows that there exist $\varepsilon_{2}, \varepsilon_{3} \rightarrow 0$ as $\varepsilon, \varepsilon_{1} \rightarrow 0$ such that $\left|u(x, t)-u_{e}(x)\right|<\varepsilon_{2}$ for all $x$ not in $\Omega_{\varepsilon}$ and all $t \geq 0, u_{\infty}(x)=\lim _{t \rightarrow \infty} u(x, t)$ exists for a.e. $x$ and satisfies $\left|u_{\infty}(x)-u_{e}(x)\right|<\varepsilon_{2}$ for $x$ not in $\Omega_{\varepsilon}$, and $f\left(u_{\infty}(x)\right)=f_{\infty}$ constant, where $\left|f_{\infty}-f_{e}\right|<\varepsilon_{3}$.

From Theorem 1 we see that a solution, which initially is uniformly close to some steady state satisfying (1.9) but not necessarily (1.8) and has the same mean, will approach the steady state, failing to minimize energy as $t$ becomes large. Of course, if the steady state is discontinuous, corresponding to a mixture of phases, the hypotheses require that the initial data have discontinuities in the same locations, so one might object that the solution is "trapped" by restricting the choice of initial data. Theorem 2 shows that this explanation is too simplistic, since there exists smooth initial data satisfying the hypotheses of Theorem 2 for any steady state $u_{e}(x)$ which satisfies (1.9), discontinuous or not. The conclusions of Theorem 2 show that such initial data yield a solution which stays arbitrarily close to the state $u_{e}(x)$ for all time, approaching some nearby discontinuous steady state asymptotically in a nonuniform fashion.

One refers to states $u$ in $\left(\alpha_{M}, \alpha_{+}\right)$or $\left(\beta_{-}, \beta_{M}\right)$ as metastable, since they are only locally energy-minimizing, and refers to states satisfying $u \leq \alpha_{M}$ or $u \geq \beta_{M}$ as absolutely stable. Theorem 2 implies that smooth solutions of (1.1) remain close to discontinuous steady states containing mixtures of metastable and absolutely stable states in arbitrary patterns. It is likely that these conclusions will be regarded as not physically correct most of the time. In that case, our results show that some other effects (interfacial energy, fluctuations, etc.) must be taken into account. What we have shown is that the dissipative influence of viscosity alone is insufficient to drive the solution to absolutely minimizing equilibrium.

The organization of this paper is as follows: In $\S 2$, we establish that equation (1.1) is well posed for initial data $u(x, 0)$ in $L_{\infty}(\Omega)$ or for continuous initial data, and we show that discontinuities in the initial data persist and that new discontinuities cannot form in finite time. We demonstrate the global existence of solutions for $t>0$ by exhibiting a positively invariant interval for $u$. In $\S 3$, we show that solutions of (1.1) stabilize to steady state. These results improve slightly a method of proof introduced in papers of Andrews and Ball $[\mathrm{AB}]$ and Pego [P], but still require certain "nondegeneracy" conditions which may not be necessary. In $\S \S 4$ and 5, we prove and discuss Theorems 1 and 2 respectively.

\section{Global existence}

In this section we construct solutions of (1.1) globally in time for initial values in $L_{\infty}(\Omega)$ (the space in which the steady state patterns of interest lie), 
and study certain regularity properties of solutions. We shall first consider a reformulation of (1.1), namely the equation

$$
u_{t}=-\nu^{-1}\left(I-(I-\nu \Delta)^{-1}\right) f(u) \stackrel{\text { def }}{=} \mathscr{F}(u) .
$$

The operator $(I-\nu \Delta)^{-1}$ is defined by setting $w=(I-\nu \Delta)^{-1} g$ when $w$ solves the problem

$$
\begin{array}{rlrl}
(I-\nu \Delta) w & =g & & \text { in } \Omega, \\
n \cdot \nabla w=0 & & \text { on } \partial \Omega .
\end{array}
$$

Theorem 2.1 (Local existence). Suppose $f$ is locally Lipschitz and $u_{0} \in X$, where the space $X$ is $L_{\infty}(\Omega)$ or $C(\bar{\Omega})$. Then there exists $T>0$ such that equation (2.1) has a unique solution $u(t)$ with $u \in C^{1}([0, T), X)$ and $u(0)=$ $u_{0}$. If $f$ is $C^{k}, 1 \leq k \leq \infty$, then $u(t) \in C^{k+1}([0, T), X)$. If $f$ is analytic, then $u \in \mathscr{H}([0, T), X)$, the space of analytic functions with values in $X$.

Proof. Equation (2.1) will be regarded as an ODE on the Banach space $X$. Theorem 2.1 follows from standard results for such ODEs (e.g. see [D]). We need to verify that the map $v \mapsto \mathscr{F}(v)$ is locally Lipschitz, $C^{k}$, or analytic on $X$ if $f$ is. But this is true provided that the linear map $g \mapsto(I-\nu \Delta)^{-1} g$ is bounded on $X$. This follows from the following more precise lemma, needed later.

Lemma 2.2. Let $g \in L_{\infty}(\Omega)$. Then problem (2.2) has a unique solution $w$ which lies in $W^{2, p}(\Omega)$ for all $p, 1<p<\infty$. If $g(x)$ is not constant a.e., then

$$
\text { ess inf } g(x)<w(x)<\operatorname{ess} \sup g(x) \text { for all } x \text { in } \Omega \text {. }
$$

Proof. The existence and regularity of $w$ follow from standard results on elliptic equations (see [T, §5.4.3]). The stated inequality is a consequence of Hopf's strong maximum principle when the solution of $(2.2)$ is $C^{2}$ [PW]. Standard embedding theorems in $[T, \S 4.6 .1]$ imply that $w \in \bar{C}^{1+\alpha}(\Omega)$ for any $\alpha<1$, the space of $C^{1}$ functions on $\Omega$ with Hölder continuous first partial derivatives. For such solutions of (2.2), the strong maximum principle is also valid; it may be proved by approximating $g$ in $(2.2)$ in $L_{p}(\Omega)$ by smooth functions.

Remark 2.3. There is nothing special about the direction of time treated in Theorem 2.1 . The solution may equally well be constructed locally for $-T<$ $t \leq 0$ as for $0 \leq t<T$.

Proposition 2.4. The solution $u(t)$ from Theorem 2.1 solves the problem (1.1)(1.3).

Proof. Define

$$
J(x, t)=f(u)+\nu u_{t} .
$$

Then from (2.1) we have

$$
J=(I-\nu \Delta)^{-1} f(u)
$$


so $J \in C\left([0, T), W^{2, p}(\Omega)\right)$ for $1<p<\infty, n \cdot \nabla J=0$ on $\partial \Omega$, and

$$
\Delta J=\nu^{-1}(\nu \Delta-I+I)(I-\nu \Delta)^{-1} f(u)=u_{t},
$$

so equation (1.1) holds.

Remark. The Sobolev embedding theorem implies that the "chemical potential" $J=f(u)+\nu u_{t}$ lies in $\bar{C}^{1+\alpha}(\Omega)$ for any $\alpha<1$ [T]. But Proposition 2.6 below shows that the terms $f(u)$ and $u_{t}$ are not necessarily more regular than $L_{\infty}(\Omega)$. Thus there is an interesting compensation of discontinuities, similar to the gain in regularity of stress seen in $[\mathrm{P}]$, producing strong solutions for rough initial data.

The following technical lemma will simplify the treatment of rough solutions of (1.1). Essentially, it allows us to treat $u(x, t)$ for a.e. $x$ as a classical solution to the ODE in (2.3). Recall that "functions" in $L_{\infty}(\Omega)$ are actually equivalence classes of bounded functions on $\Omega$, two functions being identified when they disagree only on a set of measure zero. Let $B(\Omega)$ denote the space of actual functions on $\Omega$ which are bounded, with the sup norm.

Lemma 2.5. Suppose $X=L_{\infty}(\Omega)$. Let $u(t)$ be the solution of (1.1)-(1.3) from Theorem 2.1, defined on a maximal time interval $\left(T_{1}, T_{2}\right)$. Given any bounded representative $u_{0 *}$ of the equivalence class $u_{0}$, there exists a set $\Omega_{*} \subseteq \Omega$ such that $\Omega \backslash \Omega_{*}$ has measure zero, and a function $u_{*} \in C\left(\left(T_{1}, T_{2}\right), B(\Omega)\right)$, with $u_{*}(t) \in u(t)$ for all $t \in\left(T_{1}, T_{2}\right)$, and $u_{*}(0)=u_{0 *}$ such that if $x \in \Omega_{*}$ then the function $t \mapsto u_{*}(x, t)$ is $C^{1}$ on $\left(T_{1}, T_{2}\right)$ and is a classical solution of the ODE

$$
\nu \frac{d u}{d t}(t)=-f(u(t))+J(x, t) .
$$

Proof. See the Appendix.

Proposition 2.6 (Regularity). Let $u(t)$ be the solution of (1.1)-(1.3) from Theorem 2.1, defined on a maximal time interval $\left(T_{1}, T_{2}\right)$. Take any $t \in\left(T_{1}, T_{2}\right)$, and fix $x \in \Omega_{*}$. Then:

If $u_{0}$ is continuous at $x$, then $u(\cdot, t)$ is continuous at $x$.

If $u_{0}$ is not continuous at $x$, then $u(\cdot, t)$ is not continuous at $x$.

Proof. Regarding $x$ as a parameter in equation (2.5), this result follows from standard results on continuous dependence on initial values for solutions of ODEs. Continuity at time 0 implies continuity at time $t$, and vice versa.

Now we proceed to exhibit positively invariant regions for the solution, when $f$ is as indicated in Figure 1, and is locally Lipschitz. A set $S \subseteq \mathbf{R}$ is positively invariant for the problem (1.1)-(1.3) if whenever $u_{0}(x) \in S$ for all $x \in \Omega_{*}$, it follows that $u(x, t) \in S$ for all $t \geq 0$.

Proposition 2.7 (Positively invariant regions). (1) If $S=[a, b]$ is an interval such that

$$
f(a) \leq f(u) \leq f(b) \quad \text { for all } u \in S,
$$

then $S$ is positively invariant. 
(2) If $S$ is a disjoint union of intervals $\left[a_{j}, b_{j}\right]$, such that

$$
f\left(a_{j}\right) \leq f(u) \leq f\left(b_{j}\right) \text { for all } u \in S, \text { for all } j,
$$

then $S$ is positively invariant. (It follows here that $f\left(a_{j}\right)=f\left(a_{k}\right)$ and $f\left(b_{j}\right)=$ $f\left(b_{k}\right)$ whenever $j \neq k$.)

The first part of the following lemma is needed for the proof of Proposition 2.7. These facts are also used later in the paper.

Lemma 2.8. Given any $C^{1}$ function $g: \mathbf{R} \rightarrow \mathbf{R}$, set $G(u)=\int^{u} g(f(z)) d z$. Let $u(x, t)$ be a solution of (1.1)-(1.3) defined for $0 \leq t<T_{0}$.

(1) If $g^{\prime}(z) \geq 0$ for all $z$, then the quantity $\int_{\Omega} G(u(x, t)) d x$ is nonincreasing in $t$ for $0 \leq t<T_{0}$.

(2) If $T_{0}=+\infty$ and the solution remains bounded, then

$$
\lim _{t \rightarrow \infty} \int_{\Omega} G(u(x, t)) d x
$$

exists, whatever the sign of $g^{\prime}(z)$.

Proof. Let $g$ be $C^{1}$ with $g^{\prime}(z) \geq 0$ for all $z$. Then we may compute

$$
\begin{aligned}
& \frac{d}{d t} \int_{\Omega} G(u(x, t)) d x=\int_{\Omega} g(f(u)) u_{t} \\
& \quad=-\int_{\Omega}\left[g\left(f(u)+\nu u_{t}\right)-g(f(u))\right] u_{t}+\int_{\Omega} g(J) \Delta J \\
& \quad=-\int_{\Omega} g^{\prime}(z(x, t)) \nu u_{t}^{2}-\int_{\Omega} g^{\prime}(J)|\nabla J|^{2} \leq 0 .
\end{aligned}
$$

So (1) follows. Now consider part (2). When $g^{\prime}(z) \geq 0$, the result is immediate, since $\int_{\Omega} G(u(x, t)) d x$ is decreasing and bounded below. If $G(z)$ is not monotone, set $h(z)=z+\varepsilon g(z)$. Then $\varepsilon$ may be chosen so small that $h^{\prime}(z) \geq 0$ for $|z| \leq \sup _{x, t}|u(x, t)|$. If $H^{\prime}(z)=h(f(z))$ and $F^{\prime}(z)=f(z)$, then

whence part (2) follows.

$$
\varepsilon \int_{\Omega} G(u)(t)=\int_{\Omega} H(u)(t)-\int_{\Omega} F(u)(t)+c,
$$

Proof of Proposition 2.7. We will prove part (1); part (2) is only a slight generalization. Let $f_{-}=f(a)$ and $f_{+}=f(b)$. We suppose that $f(u)<f_{-}$for $u<a$ and $f(u)>f_{+}$for $u>b$; if necessary, alter the function $f$ so this holds. Suppose $u$ is a solution of (1.1)-(1.3) for $0 \leq t<T$, with $u(x, 0) \in S$ a.e. Now, we claim that for each $t \in[0, T), u(x, t) \in[a, b]$ a.e. To prove this, choose $g(z)$ so that $g$ is $C^{1}, g(z)=0$ if $z \in\left[f_{-}, f_{+}\right], g(z)<0$ for $z<f_{-}$, and $g(z)>0$ for $z>f_{+}$. Set $G(z)=\int_{f_{-}}^{z} g(f(y)) d y$, so that $G(z)=0$ for $z \in[a, b]$ and $G(z)>0$ otherwise. Applying Lemma 2.8(1), we have $0 \leq \int_{\Omega} G(u(x, t)) d x \leq \int_{\Omega} G(u(x, 0)) d x=0$. The claim follows.

Now $f(u(x, t)) \in\left[f_{-}, f_{+}\right]$for a.e. $x$, for each $t$. From equation (2.4) and Lemma 2.2, it follows that either $f(u(x, t))$ is a.e. a constant, so that the 
solution is constant everywhere, or we must have $f_{-}<J<f_{+}$for all $x, t$. From the ODE in (2.5) it easily follows that $u(x, t) \in[a, b]$ for all $x \in \Omega_{*}$, $0 \leq t<T$.

Corollary 2.9 (Global existence). Suppose $f$ is locally Lipschitz and satisfies

$$
\liminf _{z \rightarrow-\infty} f(z)<f(u)<\limsup _{z \rightarrow \infty} f(z) \text { for all } u \in \mathbf{R} \text {. }
$$

If $u_{0} \in X$, where $X$ is $L_{\infty}(\Omega)$ or $C(\bar{\Omega})$, then there is a unique solution $u(x, t)$ to $(1.1)-(1.3)$ such that $u \in C^{1}([0, \infty), X)$.

Proof. The hypothesis on $f$ guarantees that there exist arbitrarily large positively invariant intervals. Global existence follows from standard arguments, given the a priori bound on $\|u(t)\|_{L_{\infty}}$ implied by Proposition 2.7(1) and the fact that the time $T$ in Theorem 1 may be chosen as a decreasing function of $\|u(t)\|_{L_{\infty}}$.

\section{Stabilization}

In the remainder of this paper we assume $f$ is as in Figure 1, satisfying precisely the following hypothesis:

There should exist $\alpha_{-}<\alpha_{+}<\beta_{-}<\beta_{+}$such that $f\left(\alpha_{-}\right)=$

$$
f\left(\beta_{-}\right) \stackrel{\text { def }}{=} f_{-} \text {and } f\left(\alpha_{+}\right)=f\left(\beta_{+}\right) \stackrel{\text { def }}{=} f_{+}, f \text { is strictly increasing }
$$

for $u<\alpha_{+}$and $u>\beta_{-}$, and $f$ is strictly decreasing for $\alpha_{+}<u<\beta_{-}$.

In this section we show that, under certain technical hypotheses, each solution of (1.1)-(1.3) approaches some steady state $u_{\infty}(x)$ (depending on the initial data) as $T \rightarrow \infty$. One such hypothesis is that:

$$
\begin{aligned}
& f \text { is cubic, with } f(u)=a_{1}\left(u-a_{2}\right)^{3}+a_{3}\left(u-a_{2}\right)+a_{4} \text { where } \\
& a_{1}>0 \text { and } a_{3}<0 .
\end{aligned}
$$

Stabilization will be proved when $f$ satisfies (F2) for initial data with mean concentration not equal to $a_{2}$. Stabilization can be proved for all initial data, not subject to such a condition, provided that $f$ satisfies a "nondegeneracy condition" introduced in a related problem by Andrews and Ball [AB]. For $f_{-}<r<f_{+}$, denote by $u_{i}(r), i=1,2,3$, the three roots of $f(u)=r$, satisfying

$$
f\left(u_{i}(r)\right)=r, \quad i=1,2,3, \quad u_{1}(r)<u_{2}(r)<u_{3}(r) .
$$

The nondegeneracy condition is that

There are no nonnegative constants $\mu_{i}, i=0,1,2,3$, not all zero, such that $\sum_{i=1}^{3} \mu_{i} u_{i}(r) \equiv \mu_{0}$ independent of $r$ for all $r$ in any open subinterval of $\left(f_{-}, f_{+}\right)$.

Finally, stabilization can be proved under the hypotheses of Theorem 2 stated in the Introduction (see Proposition 3.5 and $\S 5$ ). 
Theorem 3.1 (Stabilization). Suppose that $f$ is locally Lipschitz and satisfies $(\mathrm{F} 1)$, and that $u_{0} \in L_{\infty}(\Omega)$. Let $u(x, t)$ be the solution of (1.1)-(1.3) defined for all $t \geq 0$. Suppose that one of the three following conditions holds:

(i) $f$ satisfies hypothesis (F2) and $|\Omega|^{-1} \int_{\Omega} u_{0}(x) d x \neq a_{2}$.

(ii) $f$ satisfies hypothesis $(\mathrm{F} 3)$.

(iii) $\liminf _{t \rightarrow \infty} \operatorname{meas}\left\{x \in \Omega \mid u(x, t) \in\left(\alpha_{+}, \beta_{-}\right)\right\}=0$.

Then there exists $u_{\infty} \in L_{\infty}(\Omega)$ such that

$$
\lim _{t \rightarrow \infty} u(x, t)=u_{\infty}(x) \text { for a.e. } x \text { in } \Omega,
$$

and $f\left(u_{\infty}(x)\right)=$ constant .

Remarks. (1) The convergence of $u(x, t)$ need not occur uniformly (see $\S 5$ below).

(2) The meaning of (iii) is that if convergence as in (3.2) does not occur, then there exists $\mu>0$ so that for all $t$ sufficently large, the set where the solution $u(x, t)$ lies in the "spinodal" interval $\left(\alpha_{+}, \beta_{-}\right)$has measure larger than $\mu$. One might expect that this is a case of no practical significance since these states are unstable (see Remark 4.2), so that, practically speaking, all solutions converge, whether or not (F3) holds.

(3) If a function $f$ violates condition (F3), clearly one could change it a small amount (only in the spinodal interval, if desired) so the condition would be satisfied. Conversely, a small change in $u_{2}(r)$ can cause $f$ to violate condition (F3) for some small interval of $r$. Moreover, condition (F3) fails to hold in the simple cases when $f$ is piecewise linear or cubic. (For cubic $f$ as in (F2), take $\mu_{0}=3 a_{1} a_{2}, \mu_{1}=\mu_{2}=\mu_{3}=1$.) So the condition is somewhat unsatisfactory.

Our proof of 3.1 is based on the arguments of Andrews and Ball [AB], which we simplify and somewhat sharpen. We divide the proof into three lemmas. Recall that $u_{t}=\Delta J=-\nu^{-1}(f(u)-J)$, where $J(x, t)=f(u)+\nu u_{t}$. Set

$$
f_{a v}(t)=|\Omega|^{-1} \int_{\Omega} f(u(x, t)) d x=|\Omega|^{-1} \int_{\Omega} J(x, t) d x .
$$

Lemma 3.2. Under the hypotheses of Theorem 3.1, as $t \rightarrow \infty$ we have

$$
\begin{gathered}
\left\|u_{t}(\cdot, t)\right\|_{L_{2}} \rightarrow 0, \\
\left\|J(\cdot, t)-f_{\mathrm{av}}(t)\right\|_{L_{\infty}} \rightarrow 0, \\
\left\|f(u(\cdot, t))-f_{\mathrm{av}}(t)\right\|_{L_{2}} \rightarrow 0 .
\end{gathered}
$$

Proof. Property (3.6) will follow immediately from (3.4) and (3.5). Choose a primitive $F(u)=\int^{u} f(z) d z$. As in the proof of Lemma 2.8, compute

$$
\frac{d}{d t} \int_{\Omega} F(u(x, t)) d x=\int_{\Omega} f(u) u_{t}=-\int_{\Omega}\left(\nu u_{t}^{2}+|\nabla J|^{2}\right) .
$$

Since the solution $u(x, t)$ is bounded, we conclude that

$$
\nu \int_{0}^{\infty} \int_{\Omega} u_{t}^{2} d x d t \leq C
$$


Here and below, $C$ denotes a generic constant independent of $t$. Now, (3.4) follows if we can show that $h(t)=\int_{\Omega} u_{t}^{2}(x, t) d x$ is uniformly continuous for $t \in[0, \infty)$. But $\|u(\cdot, t)\|_{L_{\infty}}$ is uniformly bounded, hence from (2.1) and Lemma 2.2, $\left\|u_{t}(\cdot, t)\right\|_{L_{\infty}}$ is uniformly bounded. Then for $t>s \geq 0$, using Lemma 2.5 we have

$$
\begin{aligned}
|h(t)-h(s)| & =\left|\int_{\Omega} u_{t}^{2}(x, t)-u_{t}^{2}(x, s) d x\right| \\
& \leq C \int_{\Omega}\left|u_{t}(t)-u_{t}(s)\right|=C \int_{\Omega}\left|\Delta(I-\nu \Delta)^{-1}(f(u(t))-f(u(s)))\right| \\
& \leq C\|f(u(t))-f(u(s))\|_{L_{\infty}} \leq C\|u(t)-u(s)\|_{L_{\infty}} \leq C(t-s),
\end{aligned}
$$

which shows that $h(t)$ is uniformly Lipschitz. Hence (3.4) holds. Interpolating between $L_{2}$ and $L_{\infty}$, we also conclude that

$$
\left\|u_{t}\right\|_{L_{p}} \rightarrow 0 \text { as } t \rightarrow \infty \text { for } 2 \leq p<\infty .
$$

To establish (3.5), observe that $J(\cdot, t)-f_{\mathrm{av}}(t)$ is the unique solution of the Neumann problem for finding $w(x)$ satisfying

$$
\Delta w(x)=u_{t}(x, t) \quad \text { in } \Omega, \quad n \cdot w=0 \text { on } \partial \Omega
$$

such that $\int_{\Omega} w=0$. By the results stated in Triebel $[\mathrm{T}, \S 5.4 .5]$ on this problem, for any $p, 1<p<\infty$, there exists a constant $C$ so that

$$
\left\|J(\cdot, t)-f_{\mathrm{av}}(t)\right\|_{W^{2, p}} \leq C\left\|u_{t}\right\|_{L_{p}}
$$

When $2 p>n, W^{2, p}(\Omega)$ is continuously embedded into $L_{\infty}(\Omega)$. Then (3.5) follows.

Lemma 3.3. Under the hypotheses of Theorem 3.1, $\lim _{t \rightarrow \infty} f_{\mathrm{av}}(t)$ exists.

Proof. Suppose for contradiction that the limit does not exist. Choose $p$ and $q$ so that

$$
\liminf _{t \rightarrow \infty} f_{\mathrm{av}}(t)<p<q<\limsup _{t \rightarrow \infty} f_{\mathrm{av}}(t)
$$

and so that $f_{-}$and $f_{+}$do not lie in $[p, q]$. We shall consider the case $f_{-}<$ $p<q<f_{+}$; the other cases are easier. Recall the definition of $u_{i}(r)$ from (3.1). Choose $\varepsilon>0$ small enough so that $\left|u_{i}(r)-u_{j}(r)\right|>5 \varepsilon$ if $r \in[p, q]$ and $i \neq j$. Let $Q=\left\{t \mid f_{\mathrm{av}}(t) \in[p, q]\right\}$. Define, for $t \in Q$ and $i=1,2,3$,

$$
\begin{gathered}
S_{i}^{\varepsilon}(t)=\left\{x \in \Omega|| u(x, t)-u_{i}\left(f_{\mathrm{av}}(t)\right) \mid<\varepsilon\right\}, \\
\mu_{i}^{\varepsilon}(t)=\left|S_{i}^{\varepsilon}(t)\right|, \quad \text { the measure of } S_{i}^{\varepsilon}(t), \\
S_{0}^{\varepsilon}(t)=\Omega \backslash \bigcup_{i=1}^{3} S_{i}^{\varepsilon}(t), \quad \mu_{0}^{\varepsilon}(t)=\left|S_{0}^{\varepsilon}(t)\right| .
\end{gathered}
$$

The sets $S_{i}^{\varepsilon}(t)$ are disjoint. The proof of Lemma 3.3 breaks down into three sublemmas. 
Lemma 3.3a. $\lim _{t \rightarrow \infty, t \in Q} \sum_{i=1}^{3} \mu_{i}^{\varepsilon}(t)=|\Omega|$.

Proof. From Lemma 3.2, the set $S_{f}^{\delta}(t)=\left\{x \in \Omega|| f(x, t)-f_{\mathrm{av}}(t) \mid>\delta\right\}$ satisfies $\left|S_{f}^{\delta}(t)\right| \rightarrow 0$ as $t \rightarrow \infty, t \in Q$, for any $\delta>0$. Because $f$ is strictly piecewise monotone, we may choose $\delta>0$ so that if $\left|u-u_{i}(r)\right|>\varepsilon$, then $|f(u)-r|>\delta$ for $r \in[p, q]$. Then $S_{0}^{\varepsilon}(t) \subseteq S_{f}^{\delta}(t)$, so the lemma follows.

Lemma 3.3b. Given any $g \in C^{1}(\mathbf{R})$, let $G(u)=\int^{u} g(f(z)) d z$. Then

$$
\lim _{t \rightarrow \infty} \int_{\Omega} G(u(x, t)) d x=\lim _{\substack{t \rightarrow \infty \\ t \in Q}} \sum_{i=1}^{3} \mu_{i}^{\varepsilon}(t) G\left(u_{i}\left(f_{\mathrm{av}}(t)\right)\right) .
$$

In particular, the limit on the right-hand side exists.

Proof. The limit on the left-hand side exists by Lemma 2.8. Write

$$
\begin{aligned}
\int_{\Omega} G(u(x, t)) d x= & \int_{S_{0}^{\varepsilon}(t)} G(u)+\sum_{i=1}^{3} \int_{S_{i}^{\varepsilon}(t)} G(u)-G\left(u_{i}\left(f_{\mathrm{av}}(t)\right)\right) \\
& +\sum_{i=1}^{3} \int_{S_{i}^{\varepsilon}(t)} G\left(u_{i}\left(f_{\mathrm{av}}(t)\right)\right) \\
= & T_{0}(t)+\sum_{i=1}^{3} T_{i}(t)+\sum_{i=1}^{3} \mu_{i}^{\varepsilon}(t) G\left(u_{i}\left(f_{\mathrm{av}}(t)\right)\right) .
\end{aligned}
$$

Now $\left|T_{0}\right| \leq C \mu_{0}^{\varepsilon}(t) \rightarrow 0$ as $t \rightarrow \infty$ in $Q$. For $i=1,2,3$.

$$
\left|T_{i}\right| \leq C \int_{S_{i}^{\varepsilon}(t)}\left|u(x, t)-u_{i}\left(f_{\mathrm{av}}(t)\right)\right|
$$

where $C$ is a Lipschitz constant for $G$. The estimate of this term is a bit technical: For any $\delta, 0<\delta<\varepsilon$, we have $S_{i}^{\varepsilon}(t) \supseteq S_{i}^{\delta}(t)$, so

$$
\left|T_{i}(t)\right| \leq C\left(\delta \mu_{i}^{\delta}(t)+\varepsilon\left(\mu_{i}^{\varepsilon}(t)-\mu_{i}^{\delta}(t)\right)\right)
$$

Summing this inequality over $i=1,2,3$, and using Lemma 3.3a, we find

$$
\lim _{t \rightarrow \infty} \sum_{i=1}^{3}\left|T_{i}(t)\right| \leq C \delta \text { for any } \delta>0
$$

So in fact these terms approach zero, and Lemma $3.3 \mathrm{~b}$ is proved.

Lemma 3.3c. For each $i=1,2,3, \mu_{i}=\lim _{t \rightarrow \infty, t \in Q} \mu_{i}^{\varepsilon}(t)$ exists.

Proof. Choose two nonzero nonnegative $C^{1}$ functions $g_{-}(r)$ and $g_{+}(r)$ such that their supports satisfy $\operatorname{supp} g_{-} \subseteq\left[f_{-}, p\right]$ and $\operatorname{supp} g_{+} \subseteq\left[q, f_{+}\right]$respectively. Let $G_{ \pm}(u)=\int_{f_{-}}^{u} g_{ \pm}(f(z)) d z$. For $t \in Q$ we find that $G_{ \pm}\left(u_{i}\left(f_{\mathrm{av}}(t)\right)\right)$ is independent of $t$, and in fact, for $r$ in $[p, q]$ there exist positive constants 
$\gamma_{i}^{ \pm}, i=1,2,3$, independent of $r$, with

$$
\begin{array}{llrl}
G_{-}\left(u_{1}(r)\right) & =\gamma_{1}^{-}, & G_{+}\left(u_{1}(r)\right)=0, \\
G_{-}\left(u_{2}(r)\right)=\gamma_{1}^{-}, & G_{+}\left(u_{2}(r)\right)=\gamma_{1}^{+}+\gamma_{2}^{+}, \\
G_{-}\left(u_{3}(r)\right)=\gamma_{1}^{-}+\gamma_{2}^{-}+\gamma_{3}^{-}, & & G_{+}\left(u_{3}(r)\right)=\gamma_{1}^{+}+\gamma_{2}^{+} .
\end{array}
$$

In fact,

$$
\begin{array}{ll}
\gamma_{1}^{-}=\int_{\alpha_{-}}^{u_{1}(p)} g_{-}(f(z)) d z, & \gamma_{1}=\int_{u_{1}(q)}^{\alpha_{+}} g_{+}(f(z)) d z, \\
\gamma_{2}^{-}=\int_{u_{2}(p)}^{\beta_{-}} g_{-}(f(z)) d z, & \gamma_{2}=\int_{\alpha_{+}}^{u_{2}(q)} g_{+}(f(z)) d z, \\
\gamma_{3}^{-}=\int_{\beta_{-}}^{u_{3}(p)} g_{-}(f(z)) d z . &
\end{array}
$$

Applying Lemmas $3.3 \mathrm{a}$ and $3.3 \mathrm{~b}$ we find that

$$
\left[\begin{array}{ccc}
\gamma_{1}^{-} & \gamma_{1}^{-} & \gamma_{1}^{-}+\gamma_{2}^{-}+\gamma_{3}^{-} \\
0 & \gamma_{1}^{+}+\gamma_{2}^{+} & \gamma_{1}^{+}+\gamma_{2}^{+} \\
1 & 1 & 1
\end{array}\right]\left[\begin{array}{l}
\mu_{1}^{\varepsilon}(t) \\
\mu_{2}^{\varepsilon}(t) \\
\mu_{3}^{\varepsilon}(t)
\end{array}\right]=\left[\begin{array}{c}
G_{-}^{\infty} \\
G_{+}^{\infty} \\
|\Omega|
\end{array}\right]+o(1)
$$

as $t \rightarrow \infty$ in $Q$, where $G_{ \pm}^{\infty}=\lim _{t \rightarrow \infty} \int_{\Omega} G_{ \pm}(u(x, t)) d x$. The matrix in (3.8) is nonsingular, so Lemma $3.3 \mathrm{c}$ follows.

Proof. The next step in the proof of 3.3 is to note that Lemmas $3.3 \mathrm{c}$ and $3.3 \mathrm{~b}$ imply that for any $G$ as defined in Lemma $3.3 \mathrm{~b}$, we have

$$
\lim _{t \rightarrow \infty} \int_{\Omega} G(u(x, t)) d x=\lim _{\substack{t \rightarrow \infty \\ t \in Q}} \sum_{i=1}^{3} \mu_{i} G\left(u_{i}\left(f_{\mathrm{av}}(t)\right)\right) .
$$

In particular we find that the right-hand side must equal $\sum_{i=1}^{3} \mu_{i} G\left(u_{i}(r)\right)$ for any $r \in[p, q]$. Choosing $g(z)=1, G(u)=u$, and recalling that $\int_{\Omega} u_{t}=0$, it follows that

$$
\int_{\Omega} u_{0}(x) d x=\sum_{i=1}^{3} \mu_{i} u_{i}(r) \text { for all } r \in[p, q] .
$$

If $f$ satisfies hypothesis (F3), this immediately yields a contradiction. If hypothesis (iii) of 3.1 holds, then $\mu_{2}=0$, but $u_{1}(r)$ and $u_{3}(r)$ are increasing functions, so again a contradiction is reached. Finally, for a cubic $f$ of the form in (F2), one knows that $\sum_{i=1}^{3} u_{i}(r)=3 a_{2}$, so the possibility $\mu_{i}=|\Omega| / 3$, $\int u_{0}=a_{2}|\Omega|$ cannot be excluded. However, this is the only possible solution of (3.10) for the quantities $\mu_{i}, i=1,2,3$, and $\mu_{0}=\int u_{0}$. A way to verify this is to scale the equation $f(u)=r$ so it becomes $u^{3}-3 u=2 r$, and solve by substituting $u=z+1 / z$ to find that

$$
u_{j}(r)=2 \cos \left(\frac{1}{3} \arccos (r)+\frac{2}{3} \pi j\right), \quad j=1,2,3 \text {. }
$$


Clearly these functions have pairwise independent derivative. If (3.10) has two solutions, then by taking derivatives in (3.10) with respect to $r$, one may show that $u_{i}^{\prime}(r)$ and $u_{j}^{\prime}(r)$ must be linearly dependent for some $i \neq j$, yielding a contradiction. This finishes the proof of Lemma 3.3.

To conclude the proof of Theorem 3.1, recall that for a.e. $x \in \Omega$, the function $t \mapsto u(x, t)$ is $C^{1}$ and satisfies $\nu u_{t}=-f(u)+J(x, t)$. Let $f_{\infty}=$ $\lim _{t \rightarrow \infty} f_{\mathrm{av}}(t)$. From Lemmas 3.2 and 3.3 it follows that

$$
\nu u_{t}=-\left(f(u)-f_{\infty}\right)+e(x, t),
$$

where $|e(x, t)| \rightarrow 0$ as $t \rightarrow \infty$ uniformly for $x \in \Omega$. From the lemma below and hypothesis $(\mathrm{F} 1)$, it follows that $u(x, t)$ converges boundedly almost everywhere to an $L_{\infty}$-function $u_{\infty}(x)$ satisfying $f\left(u_{\infty}(x)\right)=f_{\infty}$ a.e. This finishes the proof of Theorem 3.1.

Lemma 3.4. Assume $f: \mathbf{R} \rightarrow \mathbf{R}$ is continuous and not constant on any open interval. Assume that $z(t) \in C^{1}(0, \infty)$ is a bounded solution of $z^{\prime}(t)=f(z(t))+$ $e(t)$, where $e(t)$ is continuous with $\lim _{t \rightarrow \infty} e(t)=0$. Then $\lim _{t \rightarrow \infty} z(t)=z_{\infty}$ exists, and $f\left(z_{\infty}\right)=0$.

Proof. Since $z(t)$ is bounded, $z_{-}=\liminf z(t)$ and $z_{+}=\limsup z(t)$ are bounded. Choose any $y \in\left[z_{-}, z_{+}\right]$. We may select two sequences, $\left\{t_{n}^{+}\right\}$and $\left\{t_{n}^{-}\right\}$, so that $t_{n}^{ \pm} \rightarrow \infty$ as $n \rightarrow \infty, z\left(t_{n}^{ \pm}\right)=y, z^{\prime}\left(t_{n}^{+}\right) \geq 0$, and $z^{\prime}\left(t_{n}^{-}\right) \leq 0$ for all $n$. Then

$$
f(y)+e\left(t_{n}^{-}\right) \leq 0 \leq f(y)+e\left(t_{n}^{+}\right) \text {for all } n,
$$

so letting $n \rightarrow \infty$ we find that $f(y)=0$. Hence $f \equiv 0$ in $\left[z_{-}, z_{+}\right]$. Our hypothesis now implies that $z_{-}=z_{+}$, so $\lim _{t \rightarrow \infty} z(t)=z_{\infty}$, exists and $f\left(z_{\infty}\right)=0$.

In the absence of hypotheses (i)-(iii) of Theorem 3.1, we are unable to prove stabilization. The following result summarizes what happens if stabilization does not occur, and will be used in the proof of Theorem 2.

Proposition 3.5. Suppose that $f$ is locally Lipschitz and satisfies hypothesis (F1), and that $u_{0} \in L_{\infty}(\Omega)$. Let $u(x, t)$ be the solution of $(1.1)-(1.3)$ defined for all $t \geq 0$. Suppose that $\lim _{t \rightarrow \infty} u(x, t)$ fails to exist for a.e. $x$ in $\Omega$. Define $f_{\mathrm{av}}(t)$ by (3.3). Then there exist $p, q$ with $f_{-}<p<q<f_{+}$such that

$$
\liminf _{t \rightarrow \infty} f_{\mathrm{av}}(t)<p<q<\limsup _{t \rightarrow \infty} f_{\mathrm{av}}(t) .
$$

Defining the set $Q$ by $Q=\left\{t \mid f_{\mathrm{av}}(t) \in[p, q]\right\}$, then for any $\varepsilon$ sufficiently small and positive,

$$
\mu_{i}=\lim _{\substack{t \rightarrow \infty \\ t \in Q}} \operatorname{meas}\left\{x \in \Omega|| u(x, t)-u_{i}\left(f_{\mathrm{av}}(t)\right) \mid<\varepsilon\right\}
$$

exists for each $i=1,2,3$, (3.10) holds, and $\mu_{1}+\mu_{2}+\mu_{3}=|\Omega|$. 


\section{Stability by Linearization}

Now we begin to ascertain which steady states of (1.1) may be possible stable asymptotic states. By the method of linearization, we show in this section that virtually any steady state $u_{e}(x)$ not anywhere in the spinodal interval $\left[\alpha_{+}, \beta_{-}\right]$ is asymptotically stable to uniformly small perturbations of zero mean, and these perturbations decay exponentially fast. Theorem 4.1 below implies Theorem 1 stated in the Introduction. Steady states lying in the spinodal on a set of positive measure are unstable (see Remark 4.2).

Theorem 4.1. Assume $f$ is $C^{1}$. Suppose $u_{e}(x)$ is a steady state solution of $(1.1)$ with $f\left(u_{e}(x)\right)=f_{e}$ a.e. Suppose $f^{\prime}\left(u_{e}(x)\right) \geq f_{0}>0$ a.e. in $\Omega$. Then there exist positive $\varepsilon, C$, and $\beta$ such that, if $\left\|u_{0}-u_{e}\right\|_{L_{\infty}}<\varepsilon$ and $\int_{\Omega}\left(u_{0}-u_{e}\right)=0$, then a solution of (1.1)-(1.3) exists globally for $t \geq 0$, with

$$
\left\|u(\cdot, t)-u_{e}\right\|_{L_{\infty}} \leq C e^{-\beta t}\left\|u_{0}-u_{e}\right\|_{L_{\infty}} .
$$

Proof. Write $v(x, t)=u(x, t)-u_{e}(x)$, so $v$ must satisfy

$$
v_{t}=B v+g(v) \text {, }
$$

where

$$
\begin{gathered}
B v=\Delta(I-\nu \Delta)^{-1}\left(f^{\prime}\left(u_{e}(x)\right) v\right), \\
g(v)=\Delta(I-\nu \Delta)^{-1}\left(f\left(v+u_{e}\right)-f\left(u_{e}\right)-f^{\prime}\left(u_{e}\right) v\right) .
\end{gathered}
$$

We wish to consider (4.1) as an ordinary differential equation on a closed subspace of $L_{\infty}(\Omega)$, the space $X=\left\{v \in L_{\infty}(\Omega) \mid \int_{\Omega} v=0\right\}$. Using Lemma 2.2, and the fact that the operator $T=\Delta(I-\nu \Delta)^{-1}=-\nu^{-1}\left(I-(I-\nu \Delta)^{-1}\right)$, one may easily check that $T$ is a bounded operator from $L_{\infty}(\Omega)$ into $X$. Then clearly $B$ is a bounded linear operator on $X$ and $g$ is a locally Lipschitz function on $X$, with

$$
\|g(v)\|_{L_{\infty}}=o\left(\|v\|_{L_{\infty}}\right) \quad \text { as }\|v\|_{L_{\infty}} \rightarrow 0 .
$$

According to standard results using the linearization method (e.g. see Henry $[\mathrm{H}$, Theorem 5.1.1]), to prove Theorem 4.1 it suffices to show that the spectrum $\sigma(B)$ on $X$ lies in a half plane $\{\lambda \in \mathbf{C} \mid \operatorname{Re} \lambda \leq-\beta\}$ for some $\beta>0$.

The proof of this has two stages. The essential spectrum of $B$, denoted $\sigma_{e}(B)$, is the complement of the set of $\lambda \in \mathbf{C}$ such that either $(\lambda-B)^{-1}$ is bounded on $X$ or $\lambda$ is an isolated eigenvalue of finite multiplicity. In the first stage, we show that $\lambda$ lies in the essential spectrum of $B$ if and only if $-\nu \lambda$ lies in the essential range of $f^{\prime}\left(u_{e}\right)$, so that $\lambda \leq-f_{0} / \nu$ for $\lambda \in \sigma_{e}(B)$. In the second stage, we show that eigenvalues of $B$ must be real and satisfy $\lambda \leq-\beta$ for some $\beta>0$. The first step is achieved using the invariance of the essential spectrum under compact perturbations (see $[\mathrm{H}$, Chapter 5, Theorem A.1] for example). The second stage employs an energy method.

We proceed with stage one. $B$ has a null eigenfunction in $L_{\infty}(\Omega)$ which we normalize, writing

$$
v_{0}(x)=f^{\prime}\left(u_{e}(x)\right)^{-1} / M\left(f^{\prime}\left(u_{e}(x)\right)^{-1}\right),
$$


where $M$ is the averaging functional defined by $M v=|\Omega|^{-1} \int_{\Omega} v(x) d x$. Then $B v_{0}=0$ and $M v_{0}=1$. Define a projection $P: L_{\infty}(\Omega) \rightarrow X$ by

$$
P v(x)=v(x)-v_{0}(x) M v \text {. }
$$

( $P$ is the complementary spectral projection for $B$ for the eigenvalue $\lambda=0$ on $L_{\infty}(\Omega)$, but we do not need to prove this for what follows.) Since $P B=B$, we may write $B=-\nu^{-1}\left(B_{0}-B_{1}\right)$, where, for $v \in X$,

$$
B_{0} v=P\left(f^{\prime}\left(u_{e}\right) v\right), \quad B_{1} v=P\left((I-\nu \Delta)^{-1} f^{\prime}\left(u_{e}\right) v\right) .
$$

Because the embedding $W^{2, p}(\Omega) \rightarrow L_{\infty}(\Omega)$ is compact if $2 p>n$, by Lemma 2.2 the operator $B_{1}$ is compact. Hence the essential spectrum of $B$ is the same as that of $-\nu^{-1} B_{0}$. On the one hand, if $\lambda$ lies in the essential range of $f^{\prime}\left(u_{e}\right)$, so that $f^{\prime}\left(u_{e}(x)\right)=\lambda$ on a set of positive measure, then clearly $\lambda$ is an eigenvalue of $B_{0}$ of infinite multiplicity, and so lies in the essential spectrum. On the other hand, if $\lambda$ does not lie in the essential range of $f^{\prime}\left(u_{e}\right)$ (which is a finite set $)$, then $\left(f^{\prime}\left(u_{e}\right)-\lambda\right)^{-1} \in L_{\infty}(\Omega)$ and an explicit formula may be written for $\left(B_{0}-\lambda\right)^{-1}$ on $X$ : For $g \in X$,

$$
\left(B_{0}-\lambda\right)^{-1} g=\frac{g}{f^{\prime}\left(u_{e}\right)-\lambda}-\frac{v_{0}}{f^{\prime}\left(u_{e}\right)-\lambda} \frac{M\left(g /\left(f^{\prime}\left(u_{e}\right)-\lambda\right)\right)}{M\left(v_{0} /\left(f^{\prime}\left(u_{e}\right)-\lambda\right)\right)} \text {. }
$$

Clearly, in this case $\left(B_{0}-\lambda\right)^{-1}$ is bounded on $X$. This finishes stage one.

Now, if $\lambda \in \sigma(B)$ but $\lambda \notin \sigma_{e}(B)$, then $\lambda$ is an isolated eigenvalue, so there exists $v \in \mathbf{C} X$ nonzero such that $(B-\lambda) v=0$. That is,

$$
-\nu^{-1}\left(I-(I-\nu \Delta)^{-1}\right) f^{\prime}\left(u_{e}\right) v-\lambda v=0
$$

or

$$
\left(f^{\prime}\left(u_{e}\right)+\lambda \nu\right) v=(I-\nu \Delta)^{-1} f^{\prime}\left(u_{e}\right) v .
$$

Then $\left(f^{\prime}\left(u_{e}\right)+\lambda \nu\right) v$ lies in $W^{2, p}$ for all $p$, and

$$
(I-\nu \Delta)\left(f^{\prime}\left(u_{e}\right)+\lambda \nu\right) v=f^{\prime}\left(u_{e}\right) v .
$$

Now multiply by $\left(f^{\prime}\left(u_{e}\right)+\bar{\lambda} \nu\right) \bar{v}$ and integrate over $\Omega$. Set $w=\left(f^{\prime}\left(u_{e}\right)+\lambda \nu\right) v$. After an integration by parts, we get

$$
\begin{aligned}
& \int_{\Omega}\left|f^{\prime}\left(u_{e}\right)+\lambda \nu\right|^{2}|v|^{2}+\nu|\nabla w|^{2} \\
& \quad=\int_{\Omega}\left|f^{\prime}\left(u_{e}\right)\right|^{2}|v|^{2}+\bar{\lambda} \nu f^{\prime}\left(u_{e}\right)|v|^{2} .
\end{aligned}
$$

Immediately we find that $\lambda$ is real, since $f^{\prime}\left(u_{e}(x)\right)>0$. Then

$$
\begin{aligned}
0 & \geq \int_{\Omega}\left[\left(f^{\prime}\left(u_{e}\right)+\lambda \nu\right)^{2}-f^{\prime}\left(u_{e}\right)^{2}-\lambda \nu f^{\prime}\left(u_{e}\right)\right]|v|^{2} \\
& =\lambda \nu \int_{\Omega}\left(f^{\prime}\left(u_{e}\right)+\lambda \nu\right)|v|^{2} .
\end{aligned}
$$

Therefore $\lambda \leq 0$. 
It now suffices to show that $\lambda=0$ is not an eigenvalue. (It can only be an isolated eigenvalue.) If $v \in X$ and $B v=0$, we have

$$
f^{\prime}\left(u_{e}\right) v=(I-\nu \Delta)^{-1}\left(f^{\prime}\left(u_{e}\right) v\right) .
$$

Set $w=f^{\prime}\left(u_{e}\right) v$. Then $w \in \operatorname{Dom}(\Delta)$ in $L_{\infty}(\Omega)$ and satisfies

$$
(I-\nu \Delta) w=w \quad \text { in } \Omega, \quad n \cdot \nabla w=0 \quad \text { on } \partial \Omega \text {. }
$$

So $\Delta w=0$. Since $\Omega$ is connected it follows that $w$ is constant. Then since $\int_{\Omega} v=0$ and $f^{\prime}\left(u_{e}\right)>0$, it follows that $v=0$.

So $\lambda=0$ is not an eigenvalue, and hence there exists $\beta>0$ so that if $\lambda \in \sigma(B)$, then $\lambda \leq-\beta$. This finishes the proof of Theorem 4.1.

Remark 4.2. If the steady state $u_{e}(x)$ lies in the spinodal interval $\left(\alpha_{+}, \beta_{-}\right)$ for $x$ in a set of positive measure, then the first stage of the proof above shows that the operator $B$ has essential spectrum in the right half plane. Hence $u_{e}$ is unstable (see $[\mathrm{H}])$.

\section{ENERGY STABILITY AND ASYMPTOTICALLY DISCONTINUOUS SOLUTIONS}

In this section we assume that $f$ is locally Lipschitz and satisfies hypothesis (F1). The functions $u_{i}(r)$ are defined in (3.1). We shall address the issue of which steady state solutions of (1.1) may arise as time asymptotic limits from continuous initial data; such data yield continuous solutions by Proposition 2.5. As in $\S 4$, we are most concerned with steady states $u_{e}(x)$ having a mixture of two phases, satisfying

$$
\begin{gathered}
f\left(u_{e}(x)\right)=f_{e} \in\left(f_{-}, f_{+}\right), \\
u_{e}(x)=u_{1}\left(f_{e}\right) \text { or } u_{3}\left(f_{3}\right) \text { for a.e. } x \in \Omega .
\end{gathered}
$$

Our result below shows that all states of this form are stable (though this does not mean asymptotically stable) to a class of perturbations which always permit the initial data to be smooth. Indeed the hypotheses of the theorem below are satisfied whenever $\left\|u_{0}-u_{e}\right\|_{L_{1}(\Omega)}$ is sufficiently small. The conclusion is that the solution $u(x, t)$ with initial values $u_{0}(x)$ does not change phase at any point, and that the mean chemical potential remains always close to $f_{e}$. With the mild additional hypothesis stated in Theorem 2 that $f$ is $C^{1}$ with $f^{\prime}(u)<0$ for $u \in\left(\alpha_{+}, \beta_{-}\right)$, stabilization can be proved without the other hypotheses of Theorem 3.1. These results can guarantee that for suitable initial data, even if the solution $u(x, t)$ is smooth, the asymptotic state $u_{\infty}(x)$ is discontinuous.

Theorem 5.1. Suppose $\left\|u_{0}\right\|_{L_{\infty}} \leq M_{0}$. Let $f_{e} \in\left(f_{-}, f_{+}\right)$be arbitrary. Now for $\varepsilon_{0}, \varepsilon_{1}$ positive and sufficiently small, if

$$
\operatorname{meas}\left\{x \in \Omega|| u_{0}(x)-u_{i}\left(f_{e}\right) \mid>\varepsilon_{1} \text { for } i=1 \text { and } 3\right\}<\varepsilon_{0},
$$

then

$$
\begin{aligned}
& \text { if } u_{0}(x)<\alpha_{+} \text {then } u(x, t)<\alpha_{+} \text {for all } t>0, \\
& \text { if } u_{0}(x)>\beta_{-} \text {then } u(x, t)>\beta_{-} \text {for all } t>0,
\end{aligned}
$$




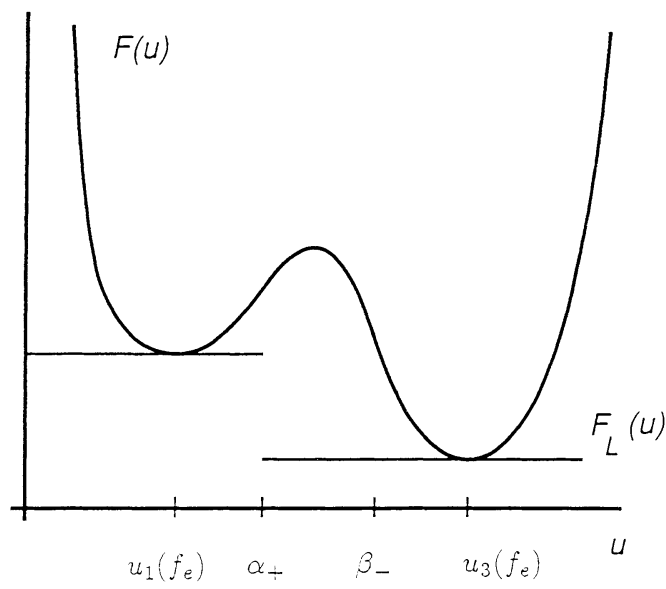

FIGURE 2

and there exists $\varepsilon_{5}\left(\varepsilon_{0}, \varepsilon_{1}\right) \rightarrow 0$ as $\varepsilon_{0}, \varepsilon_{1} \rightarrow 0$ such that

$$
\left|f_{\mathrm{av}}(t)-f_{e}\right|<\varepsilon_{5} \text { for all } t \geq 0 \text {. }
$$

Hence, if $u_{\infty}(x)=\lim _{t \rightarrow \infty} u(x, t)$ exists, with $f\left(u_{\infty}(x)\right)=f_{\infty}$ constant for a.e. $x \in \Omega$, then $\left|f_{\infty}-f_{e}\right|<\varepsilon_{5}$, and for a.e. $x \in \Omega$,

$$
\begin{aligned}
& \text { if } u_{0}(x)<\alpha_{+} \text {then } u_{\infty}(x)=u_{1}\left(f_{e}\right), \\
& \text { if } u_{0}(x)>\beta_{-} \text {then } u_{\infty}(x)=u_{3}\left(f_{e}\right) .
\end{aligned}
$$

Proof. Choose an energy function of the form $F(u)=\int^{u}\left(f(z)-f_{e}\right) d z$. Recall from Lemma 2.8 that

$$
\int_{\Omega} F(u(x, t)) d x \leq \int_{\Omega} F\left(u_{0}(x)\right) d x \text { for all } t>0 .
$$

The hypothesis $(5.2)$ means roughly that $u_{0}(x)$ is nearly everywhere near the bottom of one of the wells of $F$ (see Figure 2). In order to use (5.6) to control $u(x, t)$ when the wells of $F$ have different depths, we define a "net energy" function $E(u)$ as follows: Define an "energy floor" function $F_{L}$ by

$$
F_{L}(u)= \begin{cases}F\left(u_{1}\left(f_{e}\right)\right) & \text { if } u<\alpha_{+}, \\ F\left(u_{3}\left(f_{e}\right)\right) & \text { if } u>\beta_{-}, \\ \min F(u) & \text { if } \alpha_{+} \leq u \leq \beta_{-} .\end{cases}
$$

The "net energy" function is defined by $E(u)=F(u)-F_{L}(u)$. It satisfies

$$
\begin{array}{ll}
E(u)=0 & \text { when } u=u_{1}\left(f_{e}\right) \text { or } u_{3}\left(f_{e}\right), \\
E(u)>0 & \text { otherwise. }
\end{array}
$$

Definition. We say that the phases are invariant on $[0, T]$ if the implications below hold for a.e. $x \in \Omega$ and all $t \in[0, T]$ :

$$
\begin{aligned}
& \text { If } u_{0}(x)<\alpha_{+} \text {then } u(x, t)<\alpha_{+}, \\
& \text {If } u_{0}(x)>\beta \text { then } u(x, t)>\beta_{-} .
\end{aligned}
$$

Our proof of Theorem 5.1 consists of three preliminary observations, then an induction argument. The first observation follows immediately from the definitions above and (5.6): 
Lemma 5.2. If the phases are invariant on $[0, T]$, then $F_{L}\left(u_{0}(x)\right) \leq F_{L}(u(x, t))$ for $t \in[0, T]$ for a.e. $x$, and hence

$$
\int_{\Omega} E(u(x, t)) d x \leq \int_{\Omega} E\left(u_{0}(x)\right) d x .
$$

The hypothesis (5.2) implies that the right-hand side of (5.7) is small. In particular, the a priori bounds of Proposition 2.7 imply that for all $t>0$, we may write

$$
\|u(\cdot, t)\|_{L_{\infty}} \leq M_{u}, \quad\|f(u(\cdot, t))\|_{L_{\infty}} \leq M_{f}, \quad\|E(u(\cdot, t))\|_{L_{\infty}} \leq M_{e} .
$$

If $L$ is a Lipschitz constant for $f$ on $\left[-M_{u}, M_{u}\right]$, then for

$$
\varepsilon_{1}<\min \left\{\alpha_{+}-u_{1}\left(f_{e}\right), u_{3}\left(f_{e}\right)-\beta_{-}\right\}
$$

we find that $\left|u-u_{i}\left(f_{e}\right)\right|<\varepsilon_{1}$ for $i=1$ or 3 implies $E(u)<L \varepsilon_{1}^{2} / 2$. Then (5.2) yields

$$
\int_{\Omega} E\left(u_{0}(x)\right) d x \leq \varepsilon_{1}^{2} L|\Omega| / 2+\varepsilon_{0} M_{e} .
$$

Lemma 5.3. Define $C_{0}=\min \left\{f_{+}-f_{e}, f_{e}-f_{-}\right\}$and recall $J=\nu u_{t}+f(u)$. If

$$
\left\|J(\cdot, t)-f_{e}\right\|_{L_{\infty}}<C_{0} \text { for } t \in[0, T],
$$

then the phases are invariant on $[0, T]$.

Proof. Write $\nu u_{t}=-\left(f(u)-f_{e}\right)+\left(J-f_{e}\right)$. Recall that for a.e. $x$ in $\Omega$, $u(x, t)$ is a classical solution of this ODE. It is clear, then, that if (5.9) holds and if $u(x, t)=\alpha_{+}$, then $u_{t}(x, t)<0$. If $u(x, t)=\beta_{-}$, then $u_{t}(x, t)>0$. It follows that the phases are invariant on $[0, T]$.

Our next goal is to establish a connection between (5.8) and (5.9). Choose some $p$ satisfying $2 p>n$. Since

$$
\begin{aligned}
(I-\nu \Delta)\left(J-f_{e}\right) & =f(u)-f_{e} \quad \text { in } \Omega, \\
n \cdot \nabla\left(J-f_{e}\right) & =0 \quad \text { on } \partial \Omega,
\end{aligned}
$$

there exists $C_{1}>0$ such that

$$
\left\|J(\cdot, t)-f_{e}\right\|_{L_{\infty}} \leq c\left\|J-f_{e}\right\|_{W^{2, p}} \leq C_{1}\left\|f(u(\cdot, t))-f_{e}\right\|_{L_{p}} .
$$

For any $\varepsilon_{2}>0$, now define

$$
\mu\left(\varepsilon_{2}, t\right)=\mid\left\{x \in \Omega|| u(x, t)-u_{i}\left(f_{e}\right) \mid>\varepsilon_{2} \text { for } i=1 \text { and } 3\right\} \mid .
$$

Observe that if $|u| \leq M_{u}$, then $\left|f(u(\cdot, t))-f_{e}\right|>L \varepsilon_{2}$ implies $\left|u-u_{i}\left(f_{e}\right)\right|>\varepsilon_{2}$ for $i=1$ and 3 . Now we may estimate

$$
\left\|f(u(\cdot, t))-f_{e}\right\|_{L_{p}} \leq\left(\left(L \varepsilon_{2}\right)^{p}|\Omega|+M_{f}^{p} \mu\left(\varepsilon_{2}, t\right)\right)^{1 / p} .
$$

Again consider (5.8). According to Chebyshev's inequality, for any positive $\varepsilon_{3}, \varepsilon_{4}$, inequality $\int_{\Omega} E(u(x, t)) d x \leq \varepsilon_{3}$ implies $\left|\left\{x \in \Omega \mid E(u(x, t)) \geq \varepsilon_{4}\right\}\right| \leq$ $\varepsilon_{3} / \varepsilon_{4}$. Define

$$
\omega\left(\varepsilon_{4}\right)=\sup _{u}\left\{\left|u-u_{i}\left(f_{e}\right)\right| \mid i=1 \text { or } 3 \text { and } E(u)<\varepsilon_{4}\right\} .
$$


Then $\omega\left(\varepsilon_{4}\right) \rightarrow 0$ as $\varepsilon_{4} \rightarrow 0$ and if $\left|u-u_{i}\left(f_{e}\right)\right|>\omega\left(\varepsilon_{4}\right)$ for $i=1$ and 3 , then $E(u)>\varepsilon_{4}$. Therefore $\mu\left(\omega\left(\varepsilon_{4}\right), t\right) \leq \varepsilon_{3} / \varepsilon_{4}$. Setting $\varepsilon_{2}=\omega\left(\varepsilon_{4}\right)$, we find

$$
\left\|f(u(\cdot, t))-f_{e}\right\|_{L_{p}} \leq\left(\omega\left(\varepsilon_{4}\right)^{p} L^{p}|\Omega|+M_{f}^{p} \varepsilon_{3} / \varepsilon_{4}\right)^{1 / p} .
$$

Set $\varepsilon_{3}=\varepsilon_{4} \omega\left(\varepsilon_{4}\right)^{p}$, and $C_{2}=\left(L^{p}|\Omega|+M_{f}^{p}\right)^{1 / p}$. Then we have

Lemma 5.4. Let $\varepsilon_{4}>0$ be such that $C_{1} C_{2} \omega\left(\varepsilon_{4}\right)<C_{0}$. Then the inequality $\int_{\Omega} E(u(x, t)) d x \leq \varepsilon_{4} \omega\left(\varepsilon_{4}\right)^{p}$ implies

$$
\left\|J(\cdot, t)-f_{e}\right\|_{L_{\infty}} \leq C_{1} C_{2} \omega\left(\varepsilon_{4}\right)<C_{0} .
$$

We are ready to finish the proof of Theorem 5.1. From (5.8), it is clear that if $\varepsilon_{1}, \varepsilon_{0}$ are sufficiently small, then

$$
\int_{\Omega} E\left(u_{0}(x)\right) d x \leq \varepsilon_{4} \omega\left(\varepsilon_{4}\right)^{p} .
$$

When (5.11) holds, we claim that the set $I$ of $T \geq 0$ such that the phases are invariant on $[0, T]$ is an open and closed subset of the interval $[0, \infty)$, which will establish that the phases are invariant on $I=[0, \infty)$.

Suppose first that the phases are invariant on $[0, T-\delta]$ for any small $\delta>0$. Then from (5.11), Lemma 5.2, and because $u \in C\left([0, \infty), L_{\infty}(\Omega)\right)$,

$$
\int_{\Omega} E(u(x, t)) d x \leq \varepsilon_{4} \omega\left(\varepsilon_{4}\right)^{p} \quad \text { for all } t \in[0, T] .
$$

Then by Lemma 5.4, $\left\|J(\cdot, t)-f_{e}\right\|_{L_{\infty}}<C_{0}$ on $[0, T]$, so the phases are invariant on $[0, T]$ by Lemma 5.3. So the set $I$ is closed.

Now if the phases are invariant on $[0, T]$ for some $T \geq 0$, then as above, (5.9) holds for $t \in[0, T]$. By continuity, (5.9) must hold for $t \in[0, T+\delta]$ for some $\delta>0$. Then the phase are invariant on $[0, T+\delta]$. Hence the set $I$ is open. We conclude that $I=[0, \infty)$. Hence the phases are invariant on $[0, \infty)$, which proves (5.3). Inequality (5.4) follows from the inequality in Lemma 5.4. This finishes the proof of Theorem 5.1.

Proof of Theorem 2. It remains to show that stabilization occurs if $\varepsilon_{1}, \varepsilon_{0}$ are sufficiently small, if $f$ is $C^{1}$ with $f^{\prime}(u)<0$ for $u$ between $\alpha_{+}$and $\beta_{-}$. Suppose not. Then the conclusions of Proposition 3.5 hold. Let $\mu_{1}, \mu_{2}, \mu_{3}$, $p$, and $q$ be as defined in Proposition 3.5. From Theorem 5.1 it follows that for $\varepsilon_{1}$ sufficiently small, $\mu_{2}<\varepsilon_{0}$, and also that $p$ and $q$ lie in the interval $\left(f_{e}-\varepsilon_{5}, f_{e}+\varepsilon_{5}\right)$. Equation (3.10) implies that

$$
\mu_{1} u_{1}^{\prime}(r)+\mu_{3} u_{3}^{\prime}(r)=-\mu_{2} u_{2}^{\prime}(r) \text { for } p \leq r \leq q .
$$

Since $u_{i}^{\prime}(r)=1 / f^{\prime}\left(u_{i}(r)\right)$, our hypotheses imply that there are positive constants $C>c$ such that $\min \left(u_{1}^{\prime}(r), u_{3}^{\prime}(r)\right)>c$ and $-u_{2}^{\prime}(r)<C$. Since $\mu_{1}+\mu_{2}+\mu_{3}=|\Omega|$, equation (5.12) then implies that $\left(|\Omega|-\varepsilon_{0}\right) c<\varepsilon_{0} C$, a condition which is violated for $\varepsilon_{0}$ sufficiently small. Theorem 2 follows. 


\section{APPENDIX}

The proof of the technical Lemma 2.5 is made easy by the following representation theorem for $L_{\infty}(\Omega)$-valued continuous functions. Suppose $\Omega \subset \mathbf{R}^{n}$, and let $B(\Omega)$ be the space of actual functions on $\Omega$ which are bounded, with the sup norm. $L_{\infty}(\Omega)$ is the space of equivalence classes of functions in $B(\Omega)$ which differ on a set of measure zero.

Lemma. If $I \subset \mathbf{R}$ is an interval and $u \in C\left(I, L_{\infty}(\Omega)\right)$, then there exists $v \in$ $C(I, B(\Omega))$ such that $v(t) \in u(t)$ for all $t \in I$. Thus, for any $x \in \Omega, t \mapsto$ $v(x, t)=v(t)(x)$ is a continuous function of $t$. If two functions $v_{1}$ and $v_{2}$ have this property, then there is a fixed subset $N \subset \Omega$ of measure zero such that for all $t \in I$ and all $x \in \Omega \backslash N, v_{1}(x, t)=v_{2}(x, t)$.

Proof. Choose a dense sequence $\left\{t_{k}\right\}$ in $I$. Inductively choose $v\left(t_{k}\right) \in u\left(t_{k}\right)$ and sets $N_{k} \supset N_{k-1}$ so that $N_{k}$ has measure 0 and

$$
\sup _{x \in \Omega \backslash N_{k}}\left|v\left(t_{k}\right)(x)-v\left(t_{j}\right)(x)\right|=\left\|u\left(t_{k}\right)-u\left(t_{j}\right)\right\|_{L_{\infty}} \quad \text { for } j<k .
$$

Set $N=\bigcup N_{k}$. Then for any $t \in I$, any $x \in \Omega \backslash N$, and any subsequence $\left\{t_{k_{j}}\right\}$ converging to $t$, clearly $\left\{v\left(t_{k_{j}}\right)(x)\right\}$ is a Cauchy sequence with limit depending only on $t$. Denoting the limit by $v(t)(x)$, and setting $v(t)(x)=0$ for $x \in N$, it follows that $v \in C(I, B(\Omega))$, as desired.

To show that $v(t) \in u(t)$ for all $t \in I$, fix $t$ and choose a subsequence $\left\{t_{k_{j}}\right\}$ converging to $t$. Choosing $w \in u(t)$, there exist sets $M_{k}$ of measure zero such that

$$
\sup _{x \in \Omega \backslash M_{k}}\left|v\left(t_{k}\right)(x)-w(x)\right|=\left\|u\left(t_{k}\right)-u(t)\right\|_{L_{\infty}} \rightarrow 0 \text { as } k \rightarrow \infty .
$$

With $M=\bigcup M_{k} \cup N$, it follows that $v(t)(x)=w(x)$ for $x \in \Omega \backslash M$, so $v(t) \in u(t)$.

Supposing that two functions $v_{1}$ and $v_{2}$ have the property proved above, choose sets $N_{k}$ of measure zero such that $v_{1}\left(t_{k}\right)(x)=v_{2}\left(t_{k}\right)(x)$ for $x \in \Omega \backslash N_{k}$, and let $N=\bigcup N_{k}$. Then for all $x \in \Omega \backslash N, v_{1}\left(t_{k}\right)(x)=v_{2}\left(t_{k}\right)(x)$ for all $k$, so by continuity, $v_{1}(t)(x)=v_{2}(t)(x)$ for all $t$.

Proof of Lemma 2.5. Let $I$ be the interval $\left(T_{1}, T_{2}\right)$, and $u \in C\left(I, L_{\infty}(\Omega)\right)$ be the solution. Choose $u_{*}=v \in C(I, B(\Omega))$ according to the lemma above. We know that for all $t \in I$,

$$
u(t)=u_{0}+\nu^{-1} \int_{0}^{t}(-f(u(s))+J(\cdot, s)) d s \text { in } L_{\infty}(\Omega) .
$$

If we define $v_{1}(t)=u_{*}(t)$ and $v_{2}(t)=u_{0}+\nu^{-1} \int_{0}^{t}\left(-f\left(u_{*}(s)\right)+J(\cdot, s)\right) d s$, then $v_{1}$ and $v_{2}$ belong to $C(I, B(\Omega))$, and $v_{1}(t), v_{2}(t) \in u(t)$ for all $t \in I$. By the lemma above, there is a set $N$ of measure zero, such that for $x \in \Omega_{*}=\Omega \backslash N$,

$$
u_{*}(x, t)=u_{0}(x)+\nu^{-1} \int_{0}^{t}\left(-f\left(u_{*}(x, s)\right)+J(x, s)\right) d s
$$

which yields the result. 
Acknowledgments. The first author wishes to acknowledge the support of the Air Force Office of Scientific Research, Grant \#87-0267. The second author was partially supported by a National Science Foundation Postdoctoral Fellowship. This work was partially supported by the National Science Foundation under Grant DMS-84-01614.

\section{REFERENCES}

[A] E. C. Aifantis, On the problem of diffusion in solids, Acta Mech. 37 (1980), 265-296.

[AB] G. Andrews and J. M. Ball, Asymptotic behaviour and changes of phase in one-dimensional nonlinear viscoelasticity, J. Differential Equations 44 (1982), 306-341.

[BFJ] K. Binder, H. L. Frisch, and J. Jäckle, Kinetics of phase separation in the presence of slowly relaxing structural variables, J. Chem. Phys. 85 (1986), 1505-1512.

[C] J. W. Cahn, On spinodal decomposition, Acta Metall. 9 (1961), 795.

[CG] P. J. Chen and M. E. Gurtin, On a theory of heat conduction involving two temperatures, Z. Angew. Math. Phys. 19 (1968), 614-627.

[D] J. Dieudonné, Foundations of modern analysis, Academic Press, New York, 1969.

[Du] C. J. Dürning, Differential sorption in viscoelastic fluids, J. Polym. Sci. Polym. Phys. Ed. 23 (1985), 1831.

[H] D. Henry, Geometric theory of semilinear parabolic equations, Lecture Notes in Math. vol. 840, Springer, New York, 1981.

[JF1] J. Jäckle and H. L. Frisch, Relaxation of chemical potential and a generalized diffusion equation, J. Polym. Sci. Polym. Phys. Ed. 23 (1985), 675-682.

[JF2] _ Properties of a generalized diffusion equation with a memory, J. Chem. Phys. 85 (1986), 1621-27.

[KA] K. Kuttler and E. C. Aifantis, Existence and uniqueness in nonclassical diffusion, Quart. Appl. Math. 45 (1987), 549-560.

[P] R. L. Pego, Phase transitions in one-dimensional nonlinear viscoelasticity: admissibility and stability, Arch. Rational Mech. Anal. 97 (1987), 353-394.

[PW] M. Protter and H. Weinberger, Maximum principles in differential equations, SpringerVerlag, New York, 1984.

[S] G. B. Stephenson, Spinodal decomposition in amorphous systems, J. Non-Cryst. Sol. 66 (1984), 393-427.

[T1] T. W. Ting, Parabolic and pseudoparabolic partial differential equations, J. Math. Soc. Japan 21 (1969), 440-453.

[T2] _ _ A cooling process according to two-temperature theory of heat conductions, J. Math. Anal. Appl. 45 (1974), 23-31.

[T3] $\_$, Certain non-steady flows of second order fluids, Arch. Rational Mech. Anal. 14 (1963), $1-26$.

[T] H. Triebel, Interpolation theory, function spaces and differential operators, North-Holland, Amsterdam, 1978.

Department of Mathematics, The Technion, Haifa, Israel 32000 (Current address of A. Novick-Cohen)

Department of Mathematics, University of Michigan, Ann Arbor, Michigan 48109

Current address (R. L. Pego): Department of Mathematics, University of Maryland, College Park, Maryland 20742 